\title{
Application of the successive approximation method to the analysis of bending plates
}

\author{
Radek Gabbasov, Vladimir Filatov and Nikita Ryasny* \\ Moscow State University of Civil Engineering, Yaroslavskoe shosse, 26, Moscow, 129337, Russia
}

\begin{abstract}
This work presents an algorithm for calculating the bending plates of medium thickness according to the Reissner'. To obtain numerical results, the method of successive approximations (MSA) is used. This method has high accuracy and fast convergence, which was confirmed by the solution of a range of tasks. Publication of the results of the calculation of plates of medium thickness with the boundary conditions revised here is supposed to be in the following articles.
\end{abstract}

\section{Introduction}

One of the important sections of the theory of calculation of plates and shells is a section related to the calculation of plates of medium thickness. Below is a brief overview of the work of recent years in this area.

In [1], the development of a refined theory of plates using the method of decomposition of unknown functions in Legendre polynomials of the transverse coordinate was proposed. Isotropic plates under the action of concentrated force effects are considered. In [2] the solution for bendable rectangular continuous plates of medium thickness was obtained according to the refined theory of B. F. Vlasov. In [3], unsteady deformation of a rectangular plate of medium thickness with an oscillation damper mounted on it is investigated. In the course of formation the theory of calculation of three-layered anisotropic plates with attached masses in work [4] the assumptions for plates of medium thickness were taken into consideration within the framework of the theory of S. P. Timoshenko. The works $[5,6]$ are devoted to the formation of the numerical solution based on the finite difference method (FDM) in its classical form. In the first of these works triangular plates of medium thickness are considered, and in the second work - rectangular ones. In [7, 8] the efficiency of application of the generalized FDM equations to the calculaton of the plates of medium thickness under static and dynamic loads is shown. A simplified approach to the calculation of plates with a free edge is proposed in [9].

The difference equations of the method of successive approximations (MSA) are applied in [10-13] to the calculation of thin bending plates in the form that allows to take into consideration possible discontinuities: of the sought function, its derivatives, and the right-hand side of the initial differential equation.

\footnotetext{
Corresponding author: nikryas@gmail.com
} 
The aim of this work is to apply the difference form of MSA to the calculation of plates of medium thickness according to the Reissner's theory.

\section{Calculation Methodology}

As the initial equations, we use differential equations of plate bending, considering the influence of shear deformations and the effect of normal stress $\sigma_{z}$ [14]:

$$
\begin{gathered}
D \nabla^{4} W=q-\frac{d^{2}}{10} \frac{2-v}{1-v} \nabla^{2} q ; \\
\nabla^{4} \psi=\frac{10}{d^{2}} \psi,
\end{gathered}
$$

where

$W$ - vertical displacement of the median plane;

$\psi-$ stress function;

$q$ - intensity of a transverse load distributed according to an arbitrary function;

$d$ - plate thickness;

$v$ - Poisson's ratio;

$D$ - cylindrical rigidity.

Equations (1) and (2) can be written in the form of a system of three second-order differential equations with dimensionless variables and $P=$ const:

$$
\begin{aligned}
& \nabla^{2} m=-p ; \\
& \nabla^{2} \omega=-m ; \\
& \nabla^{2} \phi=\frac{10 a^{2}}{d^{2}} \phi,
\end{aligned}
$$

where

$$
\begin{aligned}
& w=\frac{W(x, y) D}{q_{0} a^{4}} ; \\
& p=\frac{\mathrm{q}(x, y)}{q_{0}} ; \\
& \phi=\frac{\psi(x, y)}{q_{0} a^{2}} ; \\
& \xi=\frac{x}{a} ; \\
& \eta=\frac{y}{b} ; \\
& \nabla^{2}=\frac{\partial^{2}}{\partial \xi^{2}}+\frac{\partial^{2}}{\partial \eta^{2}} ;
\end{aligned}
$$

$q_{0}$ - intensity of the distributed load at a particular point of the plate;

$a, b$ - plate dimensions.

The difference approximation in the MSA of the differential equation (3) follows as a special case from equation (2) of [12] for $\delta=\beta=\sigma=0 ; \alpha=\gamma=1$. It can be written for the rectangular mesh $\left(\tau_{1}=\tau_{2}=h_{1}=h_{2}=h\right)$ and continuous $m$ as: 


$$
\begin{aligned}
& m_{i-h, j-1}+4 m_{i-h, j}+m_{i-h, j+1}+4 m_{i, j-1}-20 m_{i, j}+4 m_{i, j+1}+m_{i+h, j-1}+4 m_{i+h, j}+ \\
& +m_{i+h, j+1}+h / 2\left(-\Delta{ }^{I-I I} m_{i, j-1}^{\xi}+7 \Delta^{I-I I} m_{i, j}^{\xi}+7 \Delta^{I I I-I V} m_{i, j}^{\xi}-\right. \\
& \left.-\Delta^{I I I-I V} m_{i, j+1}^{\xi}-\Delta^{I-I I I} m_{i-h, j}^{\eta}+7 \Delta{ }^{I-I I I} m_{i, j}^{\eta}+7 \Delta^{I I-I V} m_{i, j}^{\eta}-\Delta{ }^{I I-I V} m_{i+h, j}^{\eta}\right)= \\
& =h^{3} / 24\left(-\Delta^{I-I I} P_{i, j-1}^{\xi}+5 \Delta{ }^{I-I I} P_{i, j}^{\xi}+5 \Delta \Delta^{I I I-I V} P_{i, j}^{\xi}+\Delta^{I I I-I V} P_{i, j+1}^{\xi}+\right. \\
& \left.+{ }^{I-I I I} P_{i-h, j}^{\eta}+5 \Delta{ }^{I-I I I} P_{i, j}^{\eta}+5 \Delta{ }^{I I-I V} P_{i, j}^{\eta}+\Delta^{I I-I V} P_{i+h, j}^{\eta}\right)- \\
& -h^{2} / 12\left({ }^{I} P_{i-h, j-1}+2{ }^{I} P_{i-h, j}+2{ }^{I I I} P_{i-h, j}+{ }^{I I I} P_{i-h, j+1}+2{ }^{I} P_{i, j-1}+13{ }^{I} P_{i, j}+\right. \\
& +13{ }^{I I I} P_{i, j}+2{ }^{I I I} P_{i, j+1}+2{ }^{I I} P_{i, j-1}+13{ }^{I I} P_{i, j}+13{ }^{I V} P_{i, j}+2{ }^{I V} P_{i, j+1}+ \\
& \left.+{ }^{I I} P_{i+h, j-1}+2{ }^{I I} P_{i+h, j}+2{ }^{I V} P_{i+h, j}+{ }^{I V} P_{i+h, j+1}\right)
\end{aligned}
$$

where

$h$ - step of the mesh;

${ }_{\Delta}^{I-I I} m_{i, j}^{\xi}$ - value of the finite discontinuity of the derivative $\left(\frac{\partial m}{\partial \xi}\right)_{i j}$ on the border of the elements I-II;

other values of this kind, for example ${ }_{\Delta}^{I-I I} P_{i, j-1}^{\xi}$, have a similar meaning.

From the equation (6) taking into account (4) we obtain the difference equation with $w^{\xi \xi}=\frac{\partial^{2} w}{\partial \xi^{2}}$ and $w^{\eta \eta}=\frac{\partial^{2} w}{\partial \eta^{2}}$.

The upper left indexes in the form of Roman numerals in (6) denote the numbers of the elements that form the mesh near the point $i, j$.

To approximate (4), it is sufficient to replace $m$ and $p$ in (6) by $w$ and $m$, respectively. The difference analogue of the equation (5) is written with $m$ and $p$ replaced by $\phi$ and $\left(-\frac{10 a^{2}}{d^{2}} \phi\right)$, respectively. The difference approximation of the equation (4) can also be written using the MSA for one of the second derivatives of the sought function $w$ [10]:

$$
\begin{aligned}
& 2 w_{i-h, j-1}^{\xi \xi}+8 w_{i-h, j}^{\xi \xi}+2 w_{i-h, j+1}^{\xi \xi}+8 w_{i, j-1}^{\xi \xi}-40 w_{i, j}^{\xi \xi}+8 w_{i, j+1}^{\xi \xi}+2 w_{i+h, j-1}^{\xi \xi}+ \\
& +8 w_{i+h, j}^{\xi \xi}+2 w_{i+h, j+1}^{\xi \xi}=-h\left(\Delta^{I-I I} m_{i, j-1}^{\xi}-\Delta^{I-I I} m_{i, j}^{\xi}-\Delta^{I I I-I V} m_{i, j}^{\xi}+\right. \\
& \left.+\Delta{ }^{I I I-I V} m_{i, j+1}^{\xi}-\Delta^{I-I I I} m_{i-h, j}^{\eta}+\Delta{ }^{I-I I I} m_{i, j}^{\eta}+\Delta^{I I-I V} m_{i, j}^{\eta}-\Delta^{I I-I V} m_{i+h, j}^{\eta}\right)- \\
& -m_{i-h, j-1}-10 m_{i-h, j}-m_{i-h, j+1}+2 m_{i, j-1}+20 m_{i, j}+2 m_{i, j+1}-m_{i+h, j-1}- \\
& -10 m_{i+h, j}-m_{i+h, j+1}
\end{aligned}
$$

The presence of jumps $m_{i, j}^{\xi}$ and $m_{i, j}^{\eta}$ in equations (6) and (7) makes it possible to calculate bending plates for discontinuous loads, such as distributed along the line load and local load, without condensation the grid near these discontinuities.

The problem is formulated for the second derivative of the deflection functions $w^{\xi \xi}$, $w^{\eta \eta}$ and the stress function $\phi$, followed by the definition of $w$.

Let us consider the hinge conditions for fixing the plate at the edge parallel to the axis $\xi$ :

$$
\varphi_{\xi}=0 ; m_{\eta}=0
$$


where

$$
\begin{aligned}
& \varphi_{\xi}=\frac{5}{12} \frac{E d}{(1+v) q_{0} a} \varphi_{x} \\
& m_{\eta}=\frac{M_{y}}{q_{0} a^{2}} \\
& \varphi_{x} \text { - angle of rotation of the cross section about the x axis; } \\
& M_{y} \text { - bending moment. }
\end{aligned}
$$

In the case of hinged edges, using the well-known Reissner equation for $m_{\eta}$, we obtain:

$$
-\left(\frac{\partial^{2} w}{\partial \eta^{2}}+v \frac{\partial^{2} w}{\partial \xi^{2}}\right)+\frac{d^{2}}{5 a^{2}} \frac{\partial Q_{\eta}}{\partial \eta}-\frac{P d^{2}}{10 a^{2}} \frac{v}{1-v}=0
$$

where

$Q_{\eta}$ - transverse force.

The derivatives are replaced by the corresponding difference expressions. The equation for determining $\phi$ at the edge point i, j can be obtained using the condition $\varphi_{\xi}=0$.

In the case of the free edge of the plate, the boundary conditions are described by the following system of differential relations, which follow from the equations for $Q_{\eta}, m_{\eta}$, $m_{\xi \eta}:$

$$
\left.\begin{array}{l}
\frac{\partial m}{\partial \eta}-\frac{\partial \phi}{\partial \xi}=0 \\
-\frac{\partial^{2} w}{\partial \eta^{2}}-v \frac{\partial^{2} w}{\partial \xi^{2}}+\frac{d^{2}}{5 a^{2}} \frac{\partial Q_{\eta}}{\partial \eta}-\frac{P d^{2}}{10 a^{2}} \frac{v}{1-v}=0 \\
(1-v) \frac{\partial^{2} w}{\partial \xi \partial \eta}-\frac{d^{2}}{10 a^{2}} \frac{\partial Q_{\xi}}{\partial \eta}=0
\end{array}\right\}
$$

Differentiating expressions for $Q_{\eta}$, we obtain $\frac{\partial Q_{\eta}}{\partial \eta}=\frac{\partial^{2} m}{\partial \eta^{2}}-\frac{\partial^{2} \phi}{\partial \xi \partial \eta}$. From (3) we obtain $\frac{\partial^{2} m}{\partial \eta^{2}}=-p-\frac{\partial^{2} m}{\partial \xi^{2}}$

We use the well-known expressions:

$$
\begin{aligned}
& \frac{\partial^{2} m}{\partial \xi^{2}}=\frac{1}{h^{2}}\left(m_{i-h, j}-2 m_{i, j}+m_{i+h, j}\right) \\
& \frac{\partial^{2} \phi}{\partial \xi \partial \eta}=\frac{1}{4 h^{2}}\left(3 \phi_{i-h, j}-4 \phi_{i-h, j+1}+\phi_{i-h, j+2}-3 \phi_{i+h, j}+4 \phi_{i+h, j+1}-\phi_{i+h, j+2}\right) \\
& \frac{\partial^{2} w}{\partial \xi \partial \eta}=\frac{1}{2 h}\left(-w_{w_{i-h, j}}^{\eta}+w_{w_{i+h, j}}^{\eta}\right)
\end{aligned}
$$

The condition $Q_{\eta}=0$ implies $\frac{\partial Q_{\eta}}{\partial \xi}=0$ or $\frac{\partial^{2} m}{\partial \xi \partial \eta}-\frac{\partial^{2} \phi}{\partial \xi^{2}}=0$; this simplifies the definition of the derivative $\frac{\partial Q_{\xi}}{\partial \eta}=\frac{\partial^{2} m}{\partial \xi \partial \eta}+\frac{\partial^{2} \phi}{\partial \eta^{2}}=\frac{\partial^{2} \phi}{\partial \xi^{2}}+\frac{\partial^{2} \phi}{\partial \eta^{2}}=\frac{10 a^{2}}{d^{2}} \phi$. 
Given expressions for the derivatives allow all three conditions on the free boundary to be expressed in terms of sought functions $w^{\xi \xi}, w^{\eta \eta}, \phi$. The boundary conditions for the remaining edges $\eta=b, \xi=0, \xi=a$ are written similarly.

The equations of the compiled algorithm are solved by iterations with the transition from one calculation point to another. The Gauss-Seidel method is applied with the use, as necessary, of the upper relaxation method (to accelerate the iterative process), which gives good convergence and saves machine time.

\section{Example}

The calculations are performed for three kinds of load:

1) equally distributed over the plate;

2) distributed along the line;

3) a local load of the «cross» type, simulating a concentrated force.

As a matter of the calculation example, a square plate with a hinged support loaded over the entire area (Figure 1) is considered. The results of the calculations show the convergence of solutions for MSA with increasing number of partitions (Table 1). The table also shows the number of iterations required to obtain a solution with a given accuracy of $0.01 \%: a=b, \frac{d}{a}=0,1, p=1, v=0,3$.

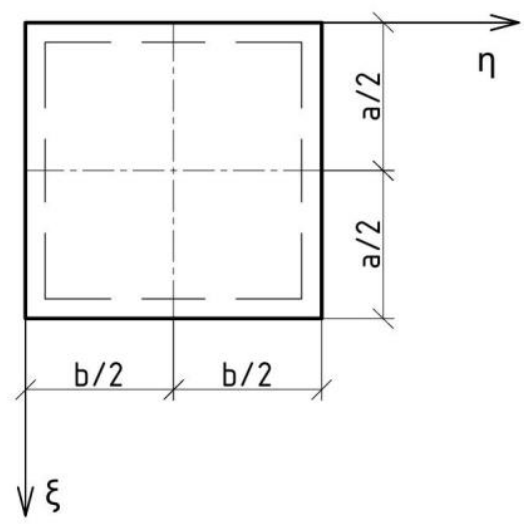

Fig. 1. Square plate with a hinged support loaded over the entire area.

Table 1. Calculation results

\begin{tabular}{|c|c|c|c|c|}
\hline $\begin{array}{c}\text { Step of the } \\
\text { mesh, h }\end{array}$ & $\begin{array}{c}\text { Number } \\
\text { of } \\
\text { iterations }\end{array}$ & $\begin{array}{c}w \\
\left(\xi=\frac{a}{2} ; \eta=\frac{b}{2}\right)\end{array}$ & $\begin{array}{c}m_{\xi} \\
\left(\xi=\frac{a}{2} ; \eta=\frac{b}{2}\right)\end{array}$ & $\begin{array}{c}Q_{\eta} \\
\left(\xi=\frac{a}{2} ; \eta=0\right)\end{array}$ \\
\hline $1 / 4$ & 45 & 0,00423 & 0,0479 & 0,3354 \\
\hline $1 / 8$ & 120 & 0,00424 & 0,0480 & 0,3377 \\
\hline $1 / 16$ & 480 & 0,00424 & 0,0480 & 0,3380 \\
\hline $\begin{array}{l}\text { Analytical } \\
\text { solution using } \\
\text { the series }\end{array}$ & - & 0,00424 & 0,0481 & 0,3370 \\
\hline
\end{tabular}

The calculation results for the first type of load are given in table 2. Comparison of analytical solutions with calculations made using the MSA reveals a coincidence of the results. 
Table 2. Hinged square plate

\begin{tabular}{|c|c|c|c|c|c|c|c|c|c|}
\hline$\frac{d}{a}$ & \multicolumn{2}{|c|}{$\begin{array}{c}\text { Analytical } \\
\text { method [15] }\end{array}$} & \multicolumn{3}{c|}{$\begin{array}{c}\text { Numerical integration } \\
{[16]}\end{array}$} & \multicolumn{2}{c|}{$\begin{array}{c}\text { FEM } \\
{[17]}\end{array}$} & \multicolumn{2}{c|}{$\begin{array}{c}\text { MSA (mesh 4x4 on a } \\
\text { quarter of the plate) }\end{array}$} \\
\hline & $w$ & $m_{\xi}$ & $w$ & $m_{\xi}$ & $Q_{\eta}$ & $w$ & $w$ & $m_{\xi}$ & $Q_{\eta}$ \\
\hline $\begin{array}{c}\text { Classical } \\
\text { theory } \\
{[18]}\end{array}$ & 0,00406 & 0,0479 & 0,00406 & 0,0479 & 0,338 & 0,00406 & 0,00406 & 0,0479 & 0,338 \\
\hline 0,01 & 0,00406 & 0,0479 & 0,00406 & 0,0479 & 0,337 & 0,00406 & 0,00406 & 0,0479 & 0,337 \\
\hline 0,05 & 0,00411 & 0,0479 & 0,00411 & 0,0479 & 0,337 & 0,00409 & 0,00410 & 0,0479 & 0,337 \\
\hline 0,10 & 0,00424 & 0,0481 & 0,00427 & 0,0479 & 0,337 & 0,00422 & 0,00424 & 0,0480 & 0,333 \\
\hline 0,20 & 0,00478 & - & 0,00490 & 0,0479 & 0,337 & 0,00475 & 0,00477 & 0,0484 & 0,339 \\
\hline 0,25 & 0,00518 & - & - & - & - & 0,00514 & 0,00515 & 0,0487 & 0,339 \\
\hline
\end{tabular}

Numerical integration in [16] is performed by segmentation method. In [19], a linear approximation and $21 \times 21$ grid on a quarter of the plate were used to calculate the plates by FEM. The cubic polynomial and the $6 \times 6$ grid (on a quarter of a plate) were used to solve the FEM in [17]. For problems of bending of jammed and hinged-supported plates, it is established that the speed of convergence and accuracy of the results of MSA exceeds FEM.

Given calculations show that the convergence of the numerical solution depends on the relative thickness of the plate $\frac{d}{a}$ : the smaller $\frac{d}{a}$, the slower the convergence and the more dense grid should be taken. Similar results are obtained in [19] for FEM, where it is also shown that for $\frac{d}{a} \leq 0,1$ the theoretical results are well confirmed by experimental ones.

On the basis of the developed algorithm, a number of engineering problems can be solved in a refined formulation in the sense of taking into account shear deformations.

\section{References}

1. I. P. Bokov, E. A. Strelnikova, Eastern-European Journal of Enterprise Technologies, Construction of fundamental solution of static equations of medium thickness isotropic plates, 4, No.7 (76), pp. 27-34 (2015)

2. A. N. Leontiev, H. A. M. Wagealla, Bulletin of MGSU, Calculation of continuous rectangular plates of medium thickness, 1, pp. 97-100 (2007)

3. O. V. Voropai, Bulletin of Kharkov National Automobile and Highway University, Simulation of unsteady deformation of a rectangular plate with an oscillation damper, 53, pp. 87-90 (2011)

4. O. A. Nekhaevskaya, Proceedings of Higher Educational Institutions. Machine Building, Obtaining static, dynamic and stability equations for three-layer plates with elastically attached masses, 10, pp. 28-33 (2011)

5. S. K. Akhmediev, G. Z. Oryntayeva, T. S. Filippova, V. Y. Teliman, Universitettin enbekteri - University works of Karaganda state technical university, Studies of the behavior of the triangular plates of thin and medium thickness, 1 (58), pp. 75-79 (2015)

6. V. A. Zhilkin, Agro-Industrial Complex of Russia, Calculation of simply supported rectangular plates using the finite difference method in MATHCAD, 24, №1, pp. 119129 (2017) 
7. R. F. Gabbasov, T. A. Hoang, Industrial and Civil Engineering, Calculation of bending plates of medium thickness using generalized equations of the finite difference method, 10, pp. 52-54 (2014)

8. R. F. Gabbasov, T. A. Hoang, Bulletin of MGSU, Calculation of bending plates of medium thickness under dynamic loads using generalized equations of the finite difference method, 10, pp. 16-23 (2014)

9. R. F. Gabbasov, V. V. Filatov, Bulletin of Civil Engineers, Approximate method of calculation of bending plates of medium thickness with a free edge, 3 (44), pp. 96-98 (2014)

10. R. F. Gabbasov, Numerical methods for solving problems of structural mechanics, Kiev: KISI, On difference forms of the method of successive approximations (1978).

11. R. F. Gabbasov, Structural Mechanics and Analysis of Constructions, Calculation of plates using the difference equations of the method of successive approximations, 3 (1980)

12. R. F. Gabbasov Applied mechanics, On difference equations in problems of strength and stability of plates, 18, No. 9 (1982)

13. R. F. Gabbasov, M. H. Ismatov, Modern methods of calculating spatial structures, MISI, The equation of the method of successive approximations for the calculation of bent plates with different boundary conditions (1987)

14. E. Reissner I. of math. a. phys., On the theory of bending of elastic plates, 23 (1944)

15. V. L. Salerno, M. A. Goldberg, Journal of applied mechanics, Effect of shear deformation on the bending and rectangular plates, 27 (1960)

16. T. Kant, Computer methods appl. mechanics and Eng., Numerical analysis of thick plates, 31, No. 1 (1982)

17. C. W. Pryor, P. M. Barber, D. Frederick, Journal of the Engineering mechanics division, ASCE, Finite element bending analysis of Reissner plates, 96, NO.EM6 (1970)

18. S. P. Timoshenko, S. Voinovsky-Krieger, Plates and shells (1966)

19. L. A. Gordon, Calculation and experimental studies of plates of medium thickness (1971) 